\title{
Current Concepts in Acute Knee Dislocation: The Missed Diagnosis?
}

\author{
Lesley McKee ${ }^{1}$, Mazin S. Ibrahim ${ }^{*}, 1$, Trevor Lawrence ${ }^{1}$, Ioannis P. Pengas ${ }^{2}$ and Wasim S. Khan ${ }^{2}$ \\ ${ }^{I}$ Trauma and Orthopaedic Department, Heart of England NHS Foundation Trust, Birmingham Heartlands Hospital, \\ Bordesley Green East, Birmingham, B9 5SS, UK \\ ${ }^{2}$ University College London Institute of Orthopaedics and Musculoskeletal Sciences, Royal National Orthopaedic \\ Hospital, Stanmore, HA7 4LP, UK
}

\begin{abstract}
Traumatic knee dislocation is a serious and potentially limb threatening injury that can be easily missed if meticulous history and examination have not been employed. Neurovascular injuries are common in this condition, and due diligence should be given to their thorough evaluation at time of secondary survey so as to avoid complications such as ischaemia, compartment syndrome and eventual amputation. There is growing evidence in the literature that morbid obesity is associated with low energy knee dislocation, therefore this should be considered when assessing this cohort of patients presenting with an acute knee injury. Early operative intervention especially with multi ligaments involvement is the preferable strategy in the management of this acute injury. Controversy exists whether to reconstruct or repair damaged structures, and whether to adopt a one stage or two stage reconstruction of the cruciate ligaments. Early rehabilitation is important and essential to achieve satisfactory outcomes. This article is an evidence-based overview of this rare but devastating injury.
\end{abstract}

Keywords: Knee dislocation, knee injuries, knee joint, ligaments, nerve injury, vascular system injuries.

\section{INTRODUCTION}

Traumatic knee dislocation (KD) is a serious and potentially limb threatening injury, albeit a relatively rare condition: the incidence has been reported as approximately $0.02-0.2 \%$ of orthopaedic injuries [1-3]. There is some variance on the definition of knee dislocation, though it can be generally considered to include disruption of at least 2 out of 6 of the major ligamentous and cartilaginous structures, with or without instability. These injuries have historically been attributed to high velocity impacts (HVKD) however more recently they are being noted in low velocity incidents (LVKD), particularly involving morbidly obese people. There is an associated high risk of neurovascular damage particularly to the popliteal artery and the common peroneal nerve, where they are tethered by the popliteal fossa boundaries and fibular head respectively, reported to be between $20-40 \%$ and as high as $64 \%$ for popliteal artery damage in some studies $[4,5]$.

Knee dislocations (KD) often reduce spontaneously out with the emergency department, potentially leading to a high rate of delayed presentation or missed diagnosis $[6,7]$. With regard to the latter, spontaneously reduced dislocations often have normal radiographs and minimal clinical signs: the physician must be vigilant and have a high index of suspicion to avoid missing the diagnosis and its potentially devastating complications. Complications of missed KD can include vascular compromise, ischaemic limb, permanent nerve damage, popliteal vessel thrombosis, acute compart-

*Address correspondence to this author at the Trauma and Orthopaedic Department, Heart of England NHS Foundation Trust, Birmingham Heartlands Hospital, Bordesley Green East, Birmingham, B9 5SS, UK;

Tel: 07902017594; E-mail: dibrm80@yahoo.com ment syndrome requiring decompression fasciiotomies and even amputation $[1,5,6,8]$.

\section{EPIDEMIOLOGY}

The true rate of knee dislocations is not known due to the number of spontaneous dislocations [8]. Knee dislocations are traditionally divided epidemiologically into those caused by high velocity trauma such as an RTA or low velocity trauma such as those sustained in sporting injuries. KD tends to occur in younger patients and occurs in a male to female ration of 4: 1 [8]. More recently there has been literature reporting the rise of knee dislocations in the morbidly obese [9-13]. Georgiadis et al. reported an increase in low velocity knee dislocations in the morbidly obese from $17 \%$ between $1995-2000$ to $53 \%$ in $2007-2012$ [11]. The same study also found that obese patients with low energy knee dislocations were more likely to have neurovascular injuries requiring surgical intervention than patients with higher energy traumatic dislocations. However traditionally, low velocity knee dislocations have lower rates of neurovascular injury [12].

\section{CLASSIFICATIONS}

Conventionally, the dislocated knee is categorised radiographically in a positional fashion by the displacement of the tibia relative to the femur. This system was originally developed by Kennedy in 1963 [2]. It classifies dislocations into 5 types: Anterior, posterior, medial, lateral or rotatory. Rotatory sub types are divided into antero-lateral, anteromedial, posterolateral and posteromedial types. Of these, posterolateral disruptions are the most difficult to reduce [6]. 
Anterior dislocations are the most common (Fig. 1A), usually occurring by forced hyperextension. They have been reported as occurring in approximately $40 \%$ of knee dislocations [1]. Kennedy's cadaveric research demonstrated that at least 30 degrees of forced hyperextension was required before an anterior dislocation occurred with the mechanism being capsular rupture followed by rupture of the anterior cruciate ligament [2]. Anterior dislocations are associated with higher likelihood of an intimal tear to the popliteal artery and therefore arterial thrombus.

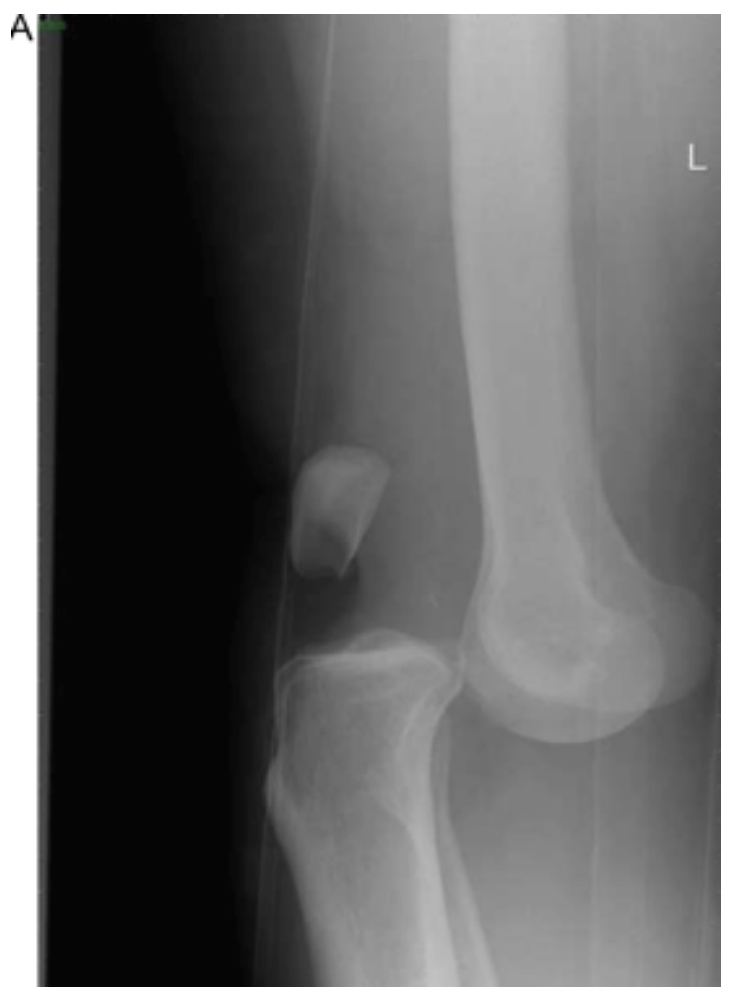

B

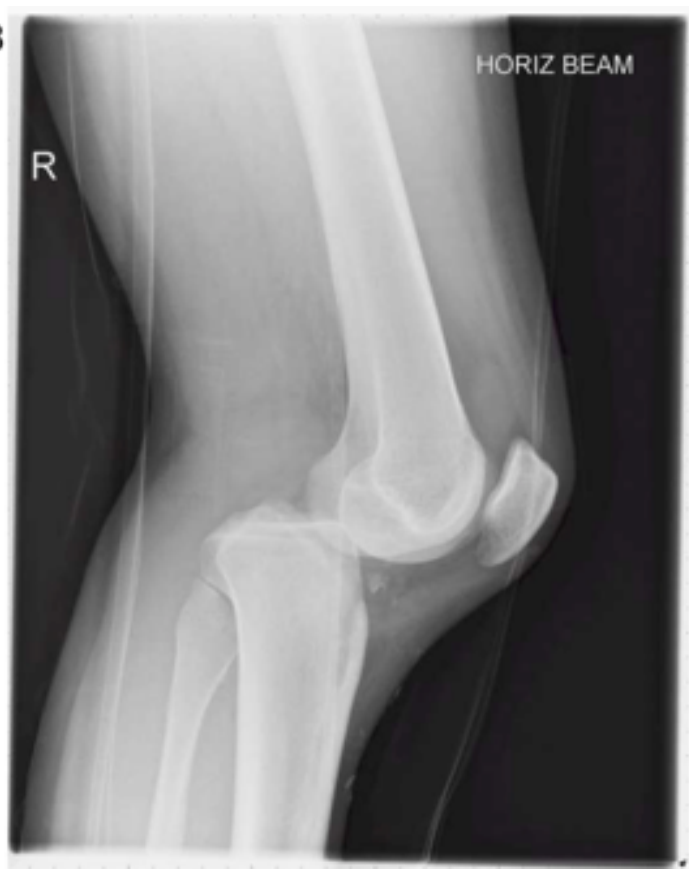

Fig. (1). Anterior (A) and posterior (B) dislocation of the tibiofemoral joint (Reproduced with permission from Howells et al. [8]).
Posterior dislocations are the second most common injury (Fig. 1B), reported as approximately 33\% of all dislocations occurring as a result of forced posterior tibial translation on the femur, classically as a result of a so-called 'dashboard injury' or during a direct fall onto a flexed knee $[1,2]$. Here the posterior cruciate ligament classically ruptures first as it is taught in knee flexion.

Medial (4\%) and lateral (18\%) dislocations are much rarer and occur due to varus/valgus stresses. They are more commonly associated with tibial plateau fractures [1]. Medial dislocations are associated with the highest risk of posterior-lateral corner (PLC) damage and thus the highest risk of irreducible dislocation [14]. A summary of these injuries can be seen in Table 1.

Subtle radiographic signs may include widened joint space, femoral epicondyle fracture, tibial plateau fracture, fibular head fracture, tibial spine fracture or a Segond sign (avulsion of the meniscotibial ligament). However due to the large number of spontaneous reductions, with either minimal or no radiographic signs, this classification system has limited practical applicability. An anatomic classification based on patterns of ligamentous damage developed by Schenck [15], and modified by Wascher [16], is now in general use and is summarised in the Table 2 . The Wascher modification added ' $C$ ' and ' $N$ ' subgroups to the Schenk classification for arterial and neurological injury respectively.

\section{ACUTE MANAGEMENT}

Following initial trauma resuscitation and treatment of life threatening injuries to standard Advanced Trauma Life Support (ATLS) management, attention should turn to the painful limb. Examination on secondary survey must pick up on both the obvious and subtle clues of acute neurovascular threat to the limb. If the knee joint is still dislocated, it is imperative that it is reduced without delay $[16,17]$. This may require sedation in the emergency department, or a formal open reduction in the operating theatre if the dislocation is non-reducible; such a situation is often associated with PLC damage. If the knee is clinically in joint, potential indicators for previous disruption include significant swelling or bruising suggesting capsular disruption; significant instability of the joint on Lachmann's testing, or instability on varus/valgus stressing of the joint, suggesting rupture of several of the major ligaments. These tests however are notoriously difficult to perform in the acutely painful joint and are potentially unreliable. A formal vascular assessment of the limb should also be performed and documented as part of the secondary survey at this time. Distal pulses may not be a reliable test as with an intimal tear of the popliteal artery, pulses may still be present $[18,19]$. Ankle brachial pressure index (ABPI) should always be performed in this instance. It has been well established that if the ABPI is 0.9 or higher the likelihood of a serious arterial injury is approaching $0 \%$ [20]. However if it is less than 0.9, there is a higher likelihood of vascular compromise and these patients should progress to duplex ultrasound or MRA imaging [20]. Angiography remains the benchmark of arterial imaging in $\mathrm{KD}$, however it is an invasive procedure and there is now evidence to suggest that the use of either selective angiography $[17,18$, 21, 22], CT angiography or MR angiography may also be 
Table 1. Summary of Kennedy's KD main subtypes.

\begin{tabular}{|c|c|c|c|c|}
\hline Direction & Frequency & Mechanism & Structures & Associated Damage \\
\hline \hline Anterior & $40 \%$ & Hyperextension & ACL rupture first & Popliteal artery (Intimal tear) \\
\hline Posterior & $33 \%$ & Dashboard & PCL rupture first & Popliteal artery (Complete tear) \\
\hline Lateral & $18 \%$ & Varus/valgus stresses & Tibial plateau fractures & Peroneal nerve \\
\hline Medial & $4 \%$ & Varus/valgus stresses & Tibial plateau fractures & PLC damage \\
\hline
\end{tabular}

Table 2. The Schenck and Wascher classifications of knee dislocations.

\begin{tabular}{|l|c|l|}
\hline Group & Sub-Group & \multicolumn{1}{|c|}{ Definition } \\
\hline \hline KD-I & & Single cruciate only \\
\hline KD-II & & Bicruciate disruption only (rare) \\
\hline KD-III & & Bicruciate and posteromedial or posterolateral disruption (common) \\
\hline KD-IV & & Bicruciate and posteromedial and posterolateral disruption \\
\hline KD-V & KD-V1 & Singlecation with associated fracture \\
\hline & KD-V2 & Bicruciate disruption only \\
\hline & KD-V3M & Bicruciate and posteromedial disruption \\
\hline & KD-V3L & Bicruciate and posterolateral disruption \\
\hline & KD-V4 & Bicruciate and posteromedial and posterolateral disruption \\
\hline & $\mathrm{C}$ & Indicates associated arterial injury when suffixed to main group \\
\hline & $\mathrm{N}$ & Indicates associated neural injury when suffixed to main group \\
\hline
\end{tabular}

appropriate [23-25]. If there is clinical evidence on examination of a devascularised limb, it has been extensively documented that patients should proceed to immediate vascular surgical intervention.

Neurological compromise associated with KD occurs most commonly to the common peroneal nerve [26] and has a reported incidence of between 10-40\% [27-29]. One study by Peskun et al. performed univarate and multivariate regression analysis to suggest that the risk of peroneal nerve injury is increased in those patients with a higher BMI ( $\mathrm{p}=$ $0.0206)$, associated fibular head fracture $(p=0.009)$ or associated vascular injury $(\mathrm{p}=0.035)$ [26]. While posing no immediate threat to limb, unlike vascular compromise, neurological injury should form part of the surgeon's decision making protocol.

\section{SURGICAL DECISION MAKING}

After the initial resuscitation, there are a number of stages in the surgical decision making process. The primary assessment to be made must be the vascular integrity of the limb. The popliteal artery is tethered superiorly at the adductor hiatus and inferiorly at the soleal muscle arcade. This relative tethering confers relatively high risk for damage [16]. The risk of popliteal artery damage has been quoted between $4.8-14 \%$ and as high as $64 \%$ in KD [30-33]. As previously discussed, frank vascular compromise requires urgent vascular surgical intervention. The restoration of arterial patency and blood supply to the limb is imperative as popliteal collateral circulation is insufficient to adequately perfuse the limb $[18,19]$. It has been documented that a delay in reperfusion of over 8 hours can increase the risk of amputation to as much as $86 \%$ [1], compared with $11 \%$ if the limb is re-perfused within 8 hours [1]. If vascular repair is required, subsequent surgical priority must be given to stabilising the knee to protect the vascular repair. This can either be in a standard above knee plaster cast or external fixator while awaiting definitive surgery [34].

Once life threatening injuries have been addressed and vascular integrity has been confirmed, surgical decision making must then proceed to several questions. These include operative versus non-operative treatment, the timing of surgical intervention and the operative techniques to be employed (reconstruction versus repair).

\section{OPERATIVE VERSUS NON-OPERATIVE INTER- VENTIONS}

Knee dislocations historically were treated in a cast or hinged brace for several weeks or months [8]. However over the last few decades, as technology has evolved, philosophy has changed in favour of operative management, to create a stable, mobile, functional knee, free from complications traditionally associated with prolonged immobilisation or non-weight bearing [35]. 
A meta-analysis in 2001 by Dedmond and Almekinders comparing operative versus non-operative management strategies found a statistically significant increase in range of motion (ROM) of 123 degrees versus 108 degrees respectively $(\mathrm{p}=<0.001)$ and post-operative Lyshom scores $(\mathrm{p}=<0.001)$ in favour of operative intervention [36]. International Knee Documentation Committee Scores (IKDC) were also found to be significantly higher in patients who were managed operatively with an average of 85.2 points versus 66.5 points $(\mathrm{p}=0.001)$ [36].

A further meta-analysis in 2011 served to build on the results of the paper by Dedmond et al. [37]. It reviewed 855 operatively managed patients and 61 non-operatively managed patients from 35 studies, and determined there was a significant difference in return to work $(p=<0.001)$ and return to sport $(p=0.001)$ rates favouring operatively managed patients. This paper also reported an improvement in functional outcome and stability with a reduction in fixed contracture when patients were managed operatively, though the review did not find these reached statistical significance. The authors did however note the poor methodological quality of several studies in its review [37].

A systematic review in 2009 by Levy et al. identified 4 studies directly comparing operative and non-operative management strategies [38]. The authors found reliably higher Lysholm scores in those patients in the operative treatment arm of the studies, although it was noted that this only achieved statistical significance in the paper by Richter et al. [29]. At a mean follow up of 51 month, Levy et al. also found higher rates of 'excellent/good' IKDC scores $(58 \% \mathrm{vs}$ $20 \%$ ) in operatively managed cohorts [38]. Levy et al. found no difference in ROM with 126 degrees vs 123 degrees mean average ROM between operative and non-operative groups, respectively.

There is no current evidence found in this review to support the utilisation of a non-operative strategy. Nevertheless, it should be remembered that most frequently, knee dislocations occur in high energy trauma situations, and the patient may well have contemporaneous injuries that pose a more significant threat to life or limb, and in these situations, the surgeon should employ their own clinical judgement.

\section{EARLY VERSUS DELAYED SURGICAL INTER- VENTION}

General consensus is now in favour of early rather than delayed surgical intervention for $\mathrm{KD}$ in order to optimise outcomes [29, 38-42]. In this context, early intervention is taken to mean no longer than 3 weeks post-injury. This allows the initial systemic inflammatory response to settle, thus reducing anaesthetic risk. Additionally it permits resolution of soft tissue swelling to allow accurate intraoperative anatomic delineation and structural repair. Three weeks has also been considered by some authors as the critical timing threshold: capsular inflammation has settled thus reducing risk of arthroscopic fluid extravasation thus facilitating an arthroscopic operation, while no significant scar tissue has yet formed [43].

In their systematic review, Levy et al. found that there were higher Lysholm scores of 90 vs 82 points and higher 'excellent/good' IKDC scores of $47 \%$ vs $31 \%$ in patients undergoing early surgical intervention, though these were not deemed statistically significant. Levy et al. found no difference (130 degrees vs 129 degrees) in ROM between the two groups when comparing early versus late intervention [38].

Early surgery (in this context taken as within 2-3 weeks following injury) would appear to be advantageous for patient outcomes. However, assuming it is clinically safe to do so, acute surgery immediately after the injury should be avoided for the documented high risk of severe arthrofibrosis [43]. This risk is however offset by the absolute indications for urgent surgery as previously discussed for an open fracture/dislocation, compartment syndrome or arterial injury [43].

With regard to the number of reconstructive surgeries following $\mathrm{KD}$, no convincing evidence currently exists in favour of either a two-stage or a single-stage operative procedure. Most surgeons would restore integrity of PCL and PLC as the matter of priority to restore stability and function to the knee. ACL reconstruction may also be performed at time of initial surgery, however due consideration must be given to procedural risk versus benefit, prolonged anaesthetic time and the potential for arthroscopic extravasation of fluid in the context of major trauma [8]. A two-stage strategy of PCL repair with collateral ligaments and soft tissues, followed by later reconstruction of the ACL if this is a functional requirement has been employed with success by some surgeons [44, 45]. More research is required to identify the optimum treatment strategy regarding the number of stages needed to reconstruct the dislocated knee.

\section{RECONSTRUCTION VERSUS REPAIR}

Surgical intervention depends both on the severity of the damage and the structures involved. Ligamentous injuries of grade 1 or 2 calibre rarely cause significant knee instability and therefore frequently do not warrant surgical intervention. This is true both in general orthopaedic context and also in KD. In principle, mid-substance tears can be reconstructed, while peripheral or avulsed lesions can be repaired [45]. The decision may be made either by guidance of MRI scan or at time of arthroscopy if an arthroscopic rather than open procedure is being performed [46]. There is no convincing evidence in the literature for reconstruction versus repair of the major ligaments [29, 41, 47]. Levy et al. found higher rates of decreased ROM, and inability to return to pre-injury activity levels with ligamentous repair as opposed to reconstruction [38]. This is supported by Stannard et al's finding of failure rate of ligamentous repair of $37 \%$, compared with a ligamentous reconstruction failure rate of $9 \%$ at a minimum follow-up of 2 years [48]. There is evidence however that PLC injuries do worse in terms of outcome following repair rather than reconstruction $[49,50]$.

With regard to neurological function, common peroneal nerve injury associated with KD may be the source of significant dysfunction and decreased quality of life [51]. Nerve injuries have been reported to resolve spontaneously in up to $50 \%$ of cases in some studies $[28,30,52,53]$ and resolution has been reported as high as $75 \%$ in some studies 
$[28,30]$. A recent study by Peskun et al. suggested that the only significant indicator $(\mathrm{p}<0.001)$ for peroneal nerve recovery was patients who were of younger age (average 22.7 years) [26].

There is a dearth of information in the literature as to the effect of surgical intervention on peroneal nerve injuries or comparison of surgical treatments for this complication, neurolysis versus tendon graft transfer for example. The same study by Peskun et al. treated the majority of their patients with nerve injury with neurolysis at the time of operative intervention for ligamentous repair. The authors considered the indication for neurolysis to be non-healing clinical peroneal nerve deficit from a traction type injury with no frank nerve transection. They reported a combined spontaneous and post-operative recovery rate of $31 \%$ [26].

\section{REHABILITATION/OUTCOMES}

Rehabilitation following traumatic KD is a gruelling and strenuous undertaking. The exact protocol will vary according to the pattern of injury and operative treatment utilised. The delay between injury and surgery of 1-3 weeks allowing the acute inflammatory reaction to subside may be put to use, and the patient may be asked to perform straight leg raises for example to prevent quadriceps muscle wasting [8]. The initial goal of post-operative rehabilitation is to protect the operative repair, particularly of the PCL, therefore some literature advocates the use of limited extension brace $[3,50,54]$. Post-operative rehabilitation is complex and beyond the scope of this review.

There is a paucity of high quality long-term outcome data in the literature. Engebretsen et al. found recently that patients with HVKD had poorer knee function across a variety of standardised measures as compared to patients sustaining LVKD [54]. However they also reported that the majority of their patients achieved fair outcomes (as measured by a median Lysholm score of 83 points) and performed normal activities regularly, as measured by a median Tegner score of 5 points at between 2 and 9 years follow-up [54].

The importance of early and repeated vascular assessments in patients with $\mathrm{KD}$ is again reiterated so as to avoid the potentially devastating consequences associated with popliteal artery damage.

\section{CONCLUSION}

Knee dislocation is a rare albeit a serious and potentially limb threatening condition. Expediency in reducing the acutely dislocated knee is vital to prevent neurovascular damage and potential for compartment syndrome and limb amputation. Missed diagnosis may be potentially disastrous. KDs may often spontaneously reduce, so in the presence of a suspicious mechanism of injury both physician and surgeon must be alert as to the possibility of knee dislocation. The present available evidence supports an operative management strategy, normally performed in the first few weeks after injury. Operative management depends on both the pattern and severity of injury, as well as the surgeon's individual skill base. Referral to a specialist centre may be appropriate, in accordance with current UK trauma management. While the majority of dislocations occur as a result of trauma, consideration to the diagnosis must be given in low energy mechanism and in seemingly trivial mechanisms of injury in the morbidly obese population. The prognosis of knee dislocations is variable and is heavily dependent on mechanism and pattern of injury.

\section{CONFLICT OF INTEREST}

The authors confirm that this article content has no conflict of interest.

\section{ACKNOWLEDGEMENTS}

Nick Howells et al. for their permission to reproduce images in their original article.

\section{REFERENCES}

[1] Green NE, Allen BL. Vascular injuries associated with dislocation of the knee. J Bone Joint Surg Am 1977; 59(2): 236-9.

[2] Kennedy JC. Complete Dislocation of the Knee Joint. J Bone Joint Surg Am 1963; 45: 889-904

[3] Chhabra A, Cha PS, Rihn JA, et al. Surgical management of knee dislocations. Surgical technique. J Bone Joint Surg Am 2005; 87Suppl 1(Pt 1): 1-21.

[4] Seroyer ST, Musahl V, Harner CD. Management of the acute knee dislocation: the Pittsburgh experience. Injury 2008; 39(7): 710-8.

[5] Merrill KD. Knee dislocations with vascular injuries. Orthop Clin North Am 1994; 25(4): 707-13.

[6] Swenson TM. Physical diagnosis of the multiple-ligament-injured knee. Clin Sports Med 2000; 19(3): 415-23.

[7] Hegyes MS, Richardson MW, Miller MD. Knee dislocation Complications of nonoperative and operative management. Clin Sports Med 2000; 19(3): 519-43.

[8] Howells NR, Brunton LR, Robinson J, Porteus AJ, Eldridge JD, Murray JR. Acute knee dislocation: an evidence based approach to the management of the multiligament injured knee. Injury 2011; 42(11): 1198-204.

[9] Hagino RT, DeCaprio JD, Valentine RJ, Clagett GP. Spontaneous popliteal vascular injury in the morbidly obese. J Vasc Surg 1998; 28(3): 458-62; discussion 62-3.

[10] Marin EL, Bifulco SS, Fast A. Obesity. A risk factor for knee dislocation. Am J Phys Med Rehabil 1990; 69(3): 132-4.

[11] Georgiadis AG, Mohammad FH, Mizerik KT, Nypaver TJ, Shepard $\mathrm{AD}$. Changing presentation of knee dislocation and vascular injury from high-energy trauma to low-energy falls in the morbidly obese. J Vasc Surg 2013; 57(5): 1196-203.

[12] Shelbourne KD, Porter DA, Clingman JA, McCarroll JR, Rettig AC. Low-velocity knee dislocation. Orthop Rev 1991; 20(11): 9951004.

[13] Folt J, Vohra T. Low-velocity knee dislocation in the morbidly obese. Am J Emerg Med 2012; 30(9): 2090.e5-6.

[14] Fanelli GC. The multiple ligament injured (dislocated) knee. J Knee Surg 2012; 25(4): 261.

[15] Schenck RC, Jr. The dislocated knee. Instrl Course Lect 1994; 43 : 127-36.

[16] Wascher DC, Dvirnak PC, DeCoster TA. Knee dislocation: initial assessment and implications for treatment. J Orthop Trauma 1997; 11(7): 525-9.

[17] Peskun CJ, Levy BA, Fanelli GC, et al. Diagnosis and management of knee dislocations. Phys Sportsmed 2010; 38(4): 101-11.

[18] Nicandri GT, Chamberlain AM, Wahl CJ. Practical management of knee dislocations: a selective angiography protocol to detect limbthreatening vascular injuries. Clin J Sport Med 2009; 19(2): 125-9.

[19] Wascher DC. High-velocity knee dislocation with vascular injury. Treatment principles. Clin Sports Med 2000; 19(3): 457-77.

[20] Mills WJ, Barei DP, McNair P. The value of the ankle-brachial index for diagnosing arterial injury after knee dislocation: a prospective study. J Trauma 2004; 56(6): 1261-5.

[21] Miranda FE, Dennis JW, Veldenz HC, Dovgan PS, Frykberg ER Confirmation of the safety and accuracy of physical examination in 
the evaluation of knee dislocation for injury of the popliteal artery: a prospective study. J Trauma 2002; 52(2): 247-51; discussion 51-

[22] Nicandri GT, Dunbar RP, Wahl CJ. Are evidence-based protocols which identify vascular injury associated with knee dislocation underutilized? Knee Surg Sports Traumatol Arthrosc 2010; 18(8): 1005-12.

[23] Peng PD, Spain DA, Tataria M, Hellinger JC, Rubin GD, Brundage SI. CT angiography effectively evaluates extremity vascular trauma. Am Surgeon 2008; 74(2): 103-7.

[24] Potter HG, Weinstein M, Allen AA, Wickiewicz TL, Helfet DL. Magnetic resonance imaging of the multiple-ligament injured knee. J Orthop Trauma 2002; 16(5): 330-9.

[25] Redmond JM, Levy BA, Dajani KA, Cass JR, Cole PA. Detecting vascular injury in lower-extremity orthopedic trauma: the role of CT angiography. Orthopedics 2008; 31(8): 761-7.

[26] Peskun CJ, Chahal J, Steinfeld ZY, Whelan DB. Risk factors for peroneal nerve injury and recovery in knee dislocation. Clin Orthop Relat Res 2012; 470(3): 774-8.

[27] Twaddle BC, Bidwell TA, Chapman JR. Knee dislocations: where are the lesions? A prospective evaluation of surgical findings in 63 cases. J Orthop Trauma 2003; 17(3): 198-202.

[28] Sisto DJ, Warren RF. Complete knee dislocation. A follow-up study of operative treatment. Clin Orthop Relat Res 1985(198): 94101

[29] Richter M, Bosch U, Wippermann B, Hofmann A, Krettek C. Comparison of surgical repair or reconstruction of the cruciate ligaments versus nonsurgical treatment in patients with traumatic knee dislocations. Am J Sports Med 2002; 30(5): 718-27.

[30] Harner CD, Waltrip RL, Bennett CH, Francis KA, Cole B, Irrgang JJ. Surgical management of knee dislocations. J Bone Joint Surg Am 2004; 86-A(2): 262-73.

[31] Rios A, Villa A, Fahandezh H, de Jose C, Vaquero J. Results after treatment of traumatic knee dislocations: a report of 26 cases. J Trauma 2003; 55(3): 489-94.

[32] McCoy GF, Hannon DG, Barr RJ, Templeton J. Vascular injury associated with low-velocity dislocations of the knee. J Bone Joint Surg Br 1987; 69(2): 285-7.

[33] Boisrenoult P, Lustig S, Bonneviale $\mathrm{P}$, et al. Vascular lesions associated with bicruciate and knee dislocation ligamentous injury. Orthop Traumatol Surg Res 2009; 95(8): 621-6.

[34] Levy BA, Krych AJ, Shah JP, Morgan JA, Stuart MJ. Staged protocol for initial management of the dislocated knee. Knee Surg Sports Traumatol Arthrosc 2010; 18(12): 1630-7.

[35] Wong CH, Tan JL, Chang HC, Khin LW, Low CO. Knee dislocations-a retrospective study comparing operative versus closed immobilization treatment outcomes. Knee Surg Sports Traumatol Arthrosc 2004; 12(6): 540-4.

[36] Dedmond BT, Almekinders LC. Operative versus nonoperative treatment of knee dislocations: a meta-analysis. [Erratum appears in Am J Knee Surg 2001 Autumn; 14(4): 220]. Am J Knee Surg 2001; 14(1): 33-8
[37] Peskun CJ, Whelan DB. Outcomes of operative and nonoperative treatment of multiligament knee injuries: an evidence-based review. Sports Med Arthrosc Rev 2011; 19(2): 167-73.

[38] Levy BA, Dajani KA, Whelan DB, et al. Decision making in the multiligament-injured knee: an evidence-based systematic review. Arthroscopy 2009; 25(4): 430-8.

[39] Mariani PP, Santoriello P, Iannone S, Condello V, Adriani E. Comparison of surgical treatments for knee dislocation. Am J Knee Surg 1999; 12(4): 214-21.

[40] Mariani PP, Margheritini F, Camillieri G. One-stage arthroscopically assisted anterior and posterior cruciate ligament reconstruction. Arthroscopy 2001; 17(7): 700-7.

[41] Liow RY, McNicholas MJ, Keating JF, Nutton RW. Ligamen repair and reconstruction in traumatic dislocation of the knee. $\mathrm{J}$ Bone Joint Surg Br 2003; 85(6): 845-51.

[42] Fanelli GC, Edson CJ. Arthroscopically assisted combined anterior and posterior cruciate ligament reconstruction in the multiple ligament injured knee: 2 - to 10 -year follow-up. Arthroscopy 2002; 18(7): 703-14.

[43] Fanelli GC. Multiple ligament-injured (dislocated) knee. Sports Med Arthrosc Rev 2011; 19(2): 81.

[44] Yeh WL, Tu YK, Su JY, Hsu RW. Knee dislocation: treatment of high-velocity knee dislocation. J Trauma 1999; 46(4): 693-701.

[45] Marks PH, Harner CD. The anterior cruciate ligament in the multiple ligament-injured knee. Clin Sports Med 1993; 12(4): 82538

[46] Cole BJ, Harner CD. The multiple ligament injured knee. Clin Sports Med 1999; 18(1): 241-62.

[47] Levy BA, Fanelli GC, Whelan DB, et al. Controversies in the treatment of knee dislocations and multiligament reconstruction. J Am Acad Orthop Surg 2009; 17(4): 197-206.

[48] Stannard JP, Brown SL, Farris RC, McGwin G, Jr., Volgas DA. The posterolateral corner of the knee: repair versus reconstruction. Am J Sports Med 2005; 33(6): 881-8.

[49] LaPrade RF. Anatomic reconstruction of the posterolateral aspect of the knee. J Knee Surg 2005; 18(2): 167-71.

[50] Larson RL. Combined instabilities of the knee. Clin Orthop Relat Res 1980(147): 68-75.

[51] Ciaramitaro P, Mondelli M, Logullo F, et al. Traumatic peripheral nerve injuries: epidemiological findings, neuropathic pain and quality of life in 158 patients. J Periph Nerv Syst 2010; 15(2): 1207.

[52] Wood MB. Peroneal nerve repair. Surgical results. Clin Orthop Relat Res 1991(267): 206-10.

[53] White J. The results of traction injuries to the common peroneal nerve. J Bone Joint Surg Br 1968; 50(2): 346-50.

[54] Engebretsen L, Risberg MA, Robertson B, Ludvigsen TC, Johansen S. Outcome after knee dislocations: a 2-9 years follow-up of 85 consecutive patients. Knee Surg Sports Traumatol Arthrosc 2009; 17(9): 1013-26. 